\title{
IN VESTIGACIÓN
}

\section{Composición en triglicéridos del aceite de las semillas en el género Coincya (Brassicaceae)}

\author{
Por J. Vioque, J. Pastor ${ }^{1}$ y E. Vioque \\ Instituto de la Grasa (CSIC), Avda. Padre García Tejero, 4. Apartado 1078, 41012 Sevilla. \\ 1Departamento de Biología Vegetal y Ecología. Facultad de Biología. Universidad de Sevilla, \\ Avda. Reina Mercedes, s/n. Apartado 1095, 41071 Sevilla.
}

\section{RESUMEN}

Composición en triglicéridos del aceite de las semillas en el género Coincya (Brassicaceae)

Se ha observado un predominio de los triglicéridos triinsaturados (entre el $81.5 \%$ y el $85.8 \%$ ). El ácido erúcico, caracteristico de las Brassicaceae, se encuentra en los triglicéridos más abundantes. Así mismo, los triglicéridos más abundantes cuentan con 3,4 ó 5 insaturaciones. En la mayoria de los taxones estudiados los principales triglicéridos son $\mathrm{C}_{22: 1} \mathrm{C}_{18: 3} \mathrm{C}_{22: 1}, \mathrm{C}_{22: 1} \mathrm{C}_{18: 2} \mathrm{C}_{22: 1}$ y $\mathrm{C}_{22: 1} \mathrm{C}_{18: 3} \mathrm{C}_{18: 3}$ posición).

PALABRAS-CLAVE: Coincya - Semilla(aceite) - Triglicéridos(com-

\section{SUMMARY}

Triglyceride composition of the seed oil in the genus Coincya (Brassicaceae).

It has been found a prevalence of triinsaturated triglycerides (between $81.5 \%$ and $85.8 \%$ ). Erucic acid, characteristic of the Brassicaceae, was found in main triglycerides. Likewise, most abundant triglycerides have 3 4 or 5 insaturations. The main ones are: $\mathrm{C}_{22: 1} \mathrm{C}_{18: 3} \mathrm{C}_{22: 1}, \mathrm{C}_{22: 1} \mathrm{C}_{18: 2} \mathrm{C}_{22: 1}$ and $\mathrm{C}_{22: 1} \mathrm{C}_{18: 3} \mathrm{C}_{18: 3}$

KEY-WORDS: Coincya - Seed (oil) - Triglycerides (composition).

\section{INTRODUCCIÓN}

El género Coincya constituye un grupo de Cruciferas silvestres con una distribución centrada en Europa Occidental y el Norte de África. Es en la Península Ibérica donde está mejor representado con 11 de los 14 taxones actualmente reconocidos en el género (1). Está incluído dentro de las Cruciferas en la tribu de las Brassiceas con otros géneros de gran importancia en la alimentación, como Brassica, Sinapis o Raphanus. A pesar del estrecho parentesco con estas plantas no tenemos constancia de que haya sido estudiado previamente por la composición en grasas. Los triglicéridos constituyen una forma fundamental de almacén de energía en plantas y animales. Influyen en las propiedades físicas, estabilidad y valor nutricional de grasas y aceites. Por otro lado, también han sido estudiados para conocer los mecanismos de absorción intestinal, detectar enfermedades por fallos en la degradación de los triglicéridos o, en el plano comercial, en análisis para la detección de aceites adulterados (2). Además, es interesante el estudio de la composición en triglicéridos para la búsqueda de nuevos aceites de plantas silvestres de nuestro pais. Por otro lado se amplía el conocimiento de nuestra flora autóctona y de sus compuestos.

En un trabajo anterior (3) se ha analizado la composición en ácidos grasos de los triglicéridos del aceite de las semillas. A partir de estos datos se ha obtenido la composición en triglicéridos en base al cálculo de probabilidades, suponiendo una distribución 1-3 al azar, 2 al azar (4).

\section{MATERIAL Y MÉTODOS}

El material se recolectó durante los años 1989, 1990 y 1991 por toda la Península Ibérica. Se tomó la precaución de que todo el material estuviera en el mismo estado de desarrollo, completamente maduro, para evitar las posibles variaciones en composición que se pueden producir hasta que se completa el desarrollo del fruto. Los testigos se encuentran depositados en el Herbario del Departamento de Biología Vegetal y Ecología de la Universidad de Sevilla (SEV).

El método seguido para la obtención de la composición en ácidos totales y en posición $\beta$ del triglicérido se detalla en un artículo anterior (5). Consiste básicamente en la extracción del aceite de la harina de las semillas con hexano, purificación de los triglicéridos por cromatografía en capa fina y transesterificación de los mismos. Los ésteres metílicos de los ácidos grasos se analizan por cromatografía gaseosa. Los $\beta$-monoglicéridos se han obtenido previamente realizando una hidrólisis enzimática con lipasa pancreática. Se ha tenido en cuenta para la composición en triglicéridos solamente los siete ácidos más abundantes: $C_{16: 0}, C_{18: 0}, C_{18: 1}, C_{18: 2}, C_{18: 3}, C_{20: 1}$ y $C_{22: 1}$.

Para el cálculo de los ácidos en posición $\alpha$ se utilizó la siguiente fórmula:

$$
\% \alpha=(3 \times \text { total }-\% \beta) / 2
$$

\section{RESULTADOS Y DISCUSIÓN}

Los triglicéridos suponen de un $80 \%$ a un $90 \%$ del extracto graso de las semillas en el género Coincya. El número de triglicéridos distintos $(\geqslant 0.1 \%)$ oscila en torno a los 100 , aunque solo unos pocos sobrepasan el $5 \%$.

Entre los más abundantes destacan los que contienen $\mathrm{C}_{22: 1}$ en posición $\alpha$ y $\mathrm{C}_{18: 1}, \mathrm{C}_{18: 2} \circ \mathrm{C}_{18: 3}$ en posición $\beta$ (Tabla I). Así, en todos los casos el principal triglicérido es $\mathrm{C}_{22: 1} \mathrm{C}_{18: 3} \mathrm{C}_{22: 1}$, con porcentajes alrededor del $10 \%$; 


\section{Tabla I. Triglicéridos más abundantes en el género Coincya}

Taxones

C. transtagana

C. longirostra

C. rupestris

subsp. rupestris

subsp.leptocarpa

C. monensis

subsp. recurvata

var. recurvata

var. granatensis

var. johnstonii

subsp. hispida

subsp. nevadensis
$C_{22: 1} C_{18: 3} C_{22: 1}=19.4 \pm 0.0$
$C_{22: 1} C_{18: 3} C_{22: 1}=11.0 \pm 0.4$

$C_{22: 1} C_{18: 3} C_{22: 1}=9.7 \pm 0.7$

$\mathrm{C}_{22: 1} \mathrm{C}_{18: 3} \mathrm{C}_{22: 1}=10.9 \pm 4.1$

$\mathrm{C}_{22: 1} \mathrm{C}_{18: 3} \mathrm{C}_{22: 1}=9.1 \pm 2.5$

$\mathrm{C}_{22: 1} \mathrm{C}_{18: 3} \mathrm{C}_{22: 1}=6.5 \pm 0.0$

$\mathrm{C}_{22: 1} \mathrm{C}_{18: 2} \mathrm{C}_{18: 2}=5.6 \pm 0.0$

$\mathrm{C}_{22: 1} \mathrm{C}_{18: 3} \mathrm{C}_{22: 1}=6.3 \pm 2.0$

$\mathrm{C}_{22: 1} \mathrm{C}_{18: 3} \mathrm{C}_{22: 1}=8.2 \pm 0.6$

$$
\begin{aligned}
& \mathrm{C}_{22: 1} \mathrm{C}_{18: 2} \mathrm{C}_{22: 1}=11.8 \pm 0.0 \\
& \mathrm{C}_{22: 1} \mathrm{C}_{18: 2} \mathrm{C}_{22: 1}=6.7 \pm 0.1 \\
& \mathrm{C}_{22: 1} \mathrm{C}_{18: 2} \mathrm{C}_{22: 1}=5.9 \pm 0.8 \\
& \mathrm{C}_{22: 1} \mathrm{C}_{18: 2} \mathrm{C}_{22: 1}=6.0 \pm 1.2 \\
& \mathrm{C}_{22: 1} \mathrm{C}_{18: 2} \mathrm{C}_{22: 1}=6.3 \pm 1.0 \\
& \mathrm{C}_{22: 1} \mathrm{C}_{18: 2} \mathrm{C}_{22: 1}=5.4 \pm 0.0 \\
& \mathrm{C}_{22: 1} \mathrm{C}_{18: 2} \mathrm{C}_{22: 1}=5.2 \pm 0.0 \\
& \mathrm{C}_{22: 1} \mathrm{C}_{18: 2} \mathrm{C}_{22: 1}=5.5 \pm 1.3 \\
& \mathrm{C}_{22: 1} \mathrm{C}_{18: 3} \mathrm{C}_{18: 1}=8.1 \pm 2.7
\end{aligned}
$$

n: número de poblaciones estudiadas.

tan solo en la var. johnstonii el más abundante es $\mathrm{C}_{22: 1} \mathrm{C}_{18: 2} \mathrm{C}_{18: 2}$ con el $5.6 \%$.

Respecto a la distribución de los triglicéridos por el tipo de ácidos que contienen (saturado o insaturado) los porcentajes más altos corresponden al grupo con tres ácidos insaturados, entre el $80 \%$ y el $90 \%$, que junto con los que tienen un ácido saturado, con valores cercanos al $15 \%$, forman prácticamente el $100 \%$ (Tabla II).

Por el contenido en moléculas de $\mathrm{C}_{22: 1}$, el grupo más

\begin{tabular}{|c|c|c|c|c|c|c|}
\hline Taxones & $\mathrm{S}_{3}$ & $I_{2}$ & $\mathrm{I}_{2} \mathrm{~S}$ & $I_{3}$ & $\mathbf{R}$ & $n$ \\
\hline C. transtagana & $0.0 \pm 0.0$ & $0.4 \pm 0.0$ & $14.7 \pm 0.0$ & $83.8 \pm 0.0$ & $1.1 \pm 0.0$ & 1 \\
\hline C. longirostra & $0.0 \pm 0.0$ & $0.5 \pm 0.2$ & $13.1 \pm 1.0$ & $85.8 \pm 1.0$ & $0.8 \pm 0.2$ & 2 \\
\hline C. rupestris & & & & & & \\
\hline subsp. rupestris & $0.0 \pm 0.0$ & $0.5 \pm 0.3$ & $15.2 \pm 1.1$ & $83.5 \pm 1.8$ & $0.9 \pm 0.4$ & 2 \\
\hline subsp. leptocarpa & $0.0 \pm 0.0$ & $0.4 \pm 0.2$ & $13.1 \pm 1.2$ & $85.6 \pm 2.3$ & $1.0 \pm 0.9$ & 2 \\
\hline $\begin{array}{l}\text { C. monensis } \\
\text { subsp. recurvata }\end{array}$ & & & & & & \\
\hline var. recurvata & $0.0 \pm 0.0$ & $0.6 \pm 0.1$ & $15.1 \pm 1.0$ & $83.4 \pm 1.3$ & $1.0 \pm 0.5$ & 8 \\
\hline var. johnstonii & $0.0 \pm 0.0$ & $0.5 \pm 0.0$ & $15.0 \pm 0.0$ & $83.6 \pm 0.0$ & $0.9 \pm 0.0$ & 1 \\
\hline var. granatensis & $0.0 \pm 0.0$ & $0.6 \pm 0.0$ & $16.8 \pm 0.0$ & $81.5 \pm 0.0$ & $1.1 \pm 0.0$ & 1 \\
\hline subsp. hispida & $0.0 \pm 0.0$ & $0.6 \pm 0.1$ & $15.8 \pm 0.8$ & $82.4 \pm 1.0$ & $1.2 \pm 0.4$ & - 6 \\
\hline subsp. nevadensis & $0.0 \pm 0.0$ & $0.8 \pm 0.1$ & $15.2 \pm 0.9$ & $83.0 \pm 0.7$ & $1.1 \pm 0.2$ & 2 \\
\hline
\end{tabular}
abundante es el representado por los triglicéridos con una sola molécula, con valores cercanos al $50 \%$, seguido de los que no tienen $\mathrm{C}_{22: 1}$ (alrededor del $30 \%$ ). En C. transtagana, debido a los altos porcentajes de $\mathrm{C}_{22: 1}$, el segundo grupo en abundancia es el que presenta dos moléculas, con el $34.5 \%$ (Tabla III).

Tabla Il. Distribución de los triglicéridos según los ácidos constituyentes sean saturados o insaturados

S: ácido saturado; I: ácido insaturado; R: resto; $\mathrm{n}$ : número de poblaciones estudiadas.
Respecto a la distribución por el número de insaturaciones los grupos con 4 y 3 son los más frecuentes, con contenidos que varían entre 20 y 25 triglicéridos distintos (Tabla IV). Sin embargo, el que representa cuantitativamente un mayor porcentaje del total es el grupo con 5 insaturaciones, en torno al $30 \%$, salvo en la var. johnstonii donde los grupos con 4 y 5 tienen porcentajes semejantes. En el resto, el grupo de 5 es seguido por los que tienen 4 o 3 insaturaciones, salvo en la subsp. leptocarpa donde los grupos más abundantes son los de 4 y 7 tras el de 5. (Tabla V).

En general, los resultados obtenidos son semejantes a los de otros taxones estudiados pertenecientes a géneros cercanos, como Brassica (6) (7).

\section{BIBLIOGRAFÍA}

1. Leadlay, E.A. and Heywood, V.H. (1990).- "The biology and systematics of the genus Coincya Porta and Rigo ex Rouy (Cruciferae)".-Bot. Jour. Linn. Soc. 102, 313-398.

2. Litchfield, C. (1972).- "Analysis of triglycerides".-1st Ed.- Academic Press., New York and London.

3. Vioque, J., Pastor, J. and Vioque, E. (1993).- "Fatty acid composition of seed oil triglycerides in Coincya (Brassicaceae).- J. Am. Oil Chemists'Soc 70, 1157-1158.

4. Christie, W.W. (1987).- "The positional distribution of fatty acids in triglycerides". En "Analysis of oils and fats". p. 313-339.- R.J. Hamilton and J.B. Rosell (Ed.), Elsevier, England.

5. Vioque, J., Pastor, J. and Vioque, E. (1990).- "Análisis de los ácidos grasos y de los esteroles de los aceites de cuatro especies de Brassica". - Lagascalia 16, 95-103.

6. Grynberg H., Ceglowska, K. and Szczepanska, H. (1966).-"Composition des glycerides de l'huile de colza".- Rev. Fr. Corps Gras 13, 595-602.

7. Vioque, J., Pastor, J. and Vioque, E. (1990).- "Análisis de la composición en triglicéridos del aceite de las semillas de algunas especies del género Brassica (Brassicaceae)".- Grasas y Aceites 41, 428-431. 
Tabla III. Distribución de los triglicéridos por los contenidos de $\mathrm{C}_{22: 1}$

\begin{tabular}{|c|c|c|c|c|c|c|}
\hline Taxones & $3 C_{22: 1}$ & $2 C_{22: 1}$ & $1 C_{22: 1}$ & $0 C_{22: 1}$ & $\mathbf{R}$ & $n$ \\
\hline C. transtagana & $0.1 \pm 0.0$ & $34.5 \pm 0.0$ & $49.0 \pm 0.0$ & $15.3 \pm 0.0$ & $1.1 \pm 0.0$ & 1 \\
\hline C. longirostra & $0.2 \pm 0.1$ & $23.8 \pm 0.5$ & $49.5 \pm 0.1$ & $25.8 \pm 0.7$ & $0.8 \pm 0.2$ & 2 \\
\hline \multicolumn{7}{|l|}{ C. rupestris } \\
\hline subsp. rupestris & $0.4 \pm 0.3$ & $23.4 \pm 1.6$ & $49.8 \pm 1.5$ & $25.6 \pm 0.8$ & $0.8 \pm 0.2$ & 2 \\
\hline subsp. leptocarpa & $0.1 \pm 0.0$ & $23.3 \pm 6.8$ & $52.3 \pm 4.0$ & $23.5 \pm 9.9$ & $1.0 \pm 0.9$ & 2 \\
\hline \multicolumn{7}{|l|}{$\begin{array}{l}\text { C. monensis } \\
\text { subsp. recurvata }\end{array}$} \\
\hline var. recurvata & $0.2 \pm 0.2$ & $21.5 \pm 3.8$ & $48.7 \pm 1.5$ & $28.6 \pm 5.3$ & $1.0 \pm 0.5$ & 8 \\
\hline var. johnstonii & $0.3 \pm 0.0$ & $15.5 \pm 0.0$ & $44.5 \pm 0.0$ & $38.8 \pm 0.0$ & $0.9 \pm 0.0$ & 1 \\
\hline var. granatensis & $0.0 \pm 0.0$ & $15.4 \pm 0.0$ & $47.9 \pm 0.0$ & $35.6 \pm 0.0$ & $1.1 \pm 0.0$ & 1 \\
\hline subsp. hispida & $0.1 \pm 0.0$ & $17.8 \pm 2.6$ & $48.8 \pm 1.4$ & $32.2 \pm 2.8$ & $1.2 \pm 0.4$ & 6 \\
\hline subsp. nevadensis & $0.2 \pm 0.1$ & $16.1 \pm 0.3$ & $44.8 \pm 3.1$ & $38.0 \pm 3.2$ & $1.1 \pm 0.2$ & 2 \\
\hline
\end{tabular}

Tabla IV. Distribución de los triglicéridos por el número de insaturaciones: número de triglicéridos distintos

\begin{tabular}{|c|c|c|c|c|c|c|c|c|c|c|}
\hline Taxones & 1 & 2 & 3 & 4 & 5 & 6 & 7 & 8 & 9 & n \\
\hline C. transtagana & $0.0 \pm 0.0$ & $7.0 \pm 0.0$ & $16 \pm 0.0$ & $18 \pm 0.0$ & $17 \pm 0.0$ & $9.0 \pm 0.0$ & $6.0 \pm 0.0$ & $2.0 \pm 0.0$ & $1.0 \pm 0.0$ & 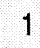 \\
\hline C. longirostra & $1.0 \pm 1.0$ & $11.5 \pm 2.5$ & $17.5 \pm 1.5$ & $20.5 \pm 0.1$ & $17.5 \pm 0.1$ & $9.5 \pm 0.1$ & $6.5 \pm 0.1$ & $2.0 \pm 0.0$ & $1.0 \pm 0.0$ & 2 \\
\hline \multicolumn{11}{|l|}{ C. rupestris } \\
\hline subsp. rupestris & $3.0 \pm 0.1$ & $12.5 \pm 2.5$ & $22.5 \pm 4.5$ & $23.0 \pm 3.0$ & $20.0 \pm 3.0$ & $11.0 \pm 1.0$ & $6.5 \pm 0.5$ & $2.0 \pm 0.0$ & $1.0 \pm 0.0$ & 2 \\
\hline subsp. leptocarpa & $1.5 \pm 0.1$ & $10.5 \pm 0.1$ & $18.5 \pm 1.5$ & $25.0 \pm 4.0$ & $19.5 \pm 0.1$ & $11.0 \pm 0.1$ & $6.0 \pm 0.0$ & $2.0 \pm 0.0$ & $1.0 \pm 0.0$ & 2 \\
\hline \multicolumn{11}{|l|}{$\begin{array}{l}\text { C. monensis } \\
\text { subsp. recurvata }\end{array}$} \\
\hline var. recurvata & $2.3 \pm 0.4$ & $12.4 \pm 1.2$ & $21.0 \pm 1.9$ & $21.3 \pm 1.9$ & $19.3 \pm 1.9$ & $10.4 \pm 0.7$ & $6.3 \pm 0.5$ & $2.0 \pm 0.0$ & $1.0 \pm 0.0$ & 0 \\
\hline var. johnstonii & $4.0 \pm 0.0$ & $16.0 \pm 0.0$ & $28.0 \pm 0.0$ & $32.0 \pm 0.0$ & $23.0 \pm 0.0$ & $10.0 \pm 0.0$ & $6.0 \pm 0.0$ & $2.0 \pm 0.0$ & $1.0 \pm 0.0$ & 1 \\
\hline var. granatensis & $3.0 \pm 0.0$ & $25.0 \pm 0.0$ & $20.0 \pm 0.0$ & $23.0 \pm 0.0$ & $18.0 \pm 0.0$ & $11.0 \pm 0.0$ & $6.0 \pm 0.0$ & $2.0 \pm 0.0$ & $1.0 \pm 0.0$ & 1 \\
\hline subsp. hispida & $2.2 \pm 0.4$ & $11.2 \pm 1.2$ & $20.8 \pm 2.0$ & $21.2 \pm 1.8$ & $19.2 \pm 1.9$ & $10.5 \pm 1.1$ & $6.2 \pm 0.4$ & $2.2 \pm 0.4$ & $1.0 \pm 0.0$ & 6 \\
\hline subsp. nevadensis & $2.0 \pm 0.0$ & $15.5 \pm 3.5$ & $24.0 \pm 6.0$ & $22.0 \pm 2.0$ & $18.0 \pm 2.0$ & $10.0 \pm 0.0$ & $5.5 \pm 0.5$ & $2.5 \pm 0.5$ & $1.0 \pm 0.0$ & 2 \\
\hline
\end{tabular}

\section{Tabla V. Distribución de los triglicéridos por el número de insaturaciones: porcentaje que aportan cada grupo}

\begin{tabular}{|c|c|c|c|c|c|c|c|c|c|c|}
\hline Taxones & 1 & 2 & 3 & 4 & 5 & 6 & 7 & 8 & 9 & $n$ \\
\hline C. transtagana & $0.0 \pm 0.0$ & $1.7 \pm 0.0$ & $11.8 \pm 0.0$ & $27.7 \pm 0.0$ & $37.8 \pm 0.0$ & $10.1 \pm 0.0$ & $8.4 \pm 0.0$ & $0.9 \pm 0.0$ & $0.5 \pm 0.0$ & 1 \\
\hline C. Iongirostra & $0.1 \pm 0.1$ & $3.9 \pm 1.8$ & $12.7 \pm 0.8$ & $21.5 \pm 0.9$ & $31.8 \pm 0.4$ & $11.5 \pm 0.7$ & $14.5 \pm 3.1$ & $1.9 \pm 0.3$ & $1.5 \pm 0.4$ & 2 \\
\hline \multicolumn{11}{|l|}{ C. rupestris } \\
\hline subsp. rupestris & $0.4 \pm 0.2$ & $3.8 \pm 1.1$ & $14.4 \pm 0.5$ & $21.1 \pm 1.8$ & $30.4 \pm 1.1$ & $13.6 \pm 0.3$ & $12.2 \pm 0.7$ & $2.1 \pm 0.2$ & $1.4 \pm 0.3$ & 2 \\
\hline subsp. leptocarpa & $0.2 \pm 0.1$ & $2.9 \pm 0.0$ & $8.8 \pm 1.6$ & $18.6 \pm 0.4$ & $30.5 \pm 3.6$ & $15.8 \pm 4.5$ & $17.9 \pm 4.6$ & $3.0 \pm 1.2$ & $1.6 \pm 0.3$ & 2 \\
\hline \multicolumn{11}{|l|}{$\begin{array}{l}\text { C. monensis } \\
\text { subsp. recurvata }\end{array}$} \\
\hline var. recurvata & $0.2 \pm 0.0$ & $3.4 \pm 0.5$ & $15.9 \pm 1.0$ & $23.9 \pm 1.4$ & $30.4 \pm 2.9$ & $12.6 \pm 1.2$ & $10.0 \pm 1.5$ & $1.7 \pm 0.6$ & $0.9 \pm 0.4$ & 8 \\
\hline var. johnstonii & $0.6 \pm 0.0$ & $3.8 \pm 0.0$ & $15.4 \pm 0.0$ & $30.0 \pm 0.0$ & $27.8 \pm 0.0$ & $10.3 \pm 0.0$ & $8.0 \pm 0.0$ & $2.0 \pm 0.0$ & $0.4 \pm 0.0$ & 1 \\
\hline var. granatensis & $0.3 \pm 0.0$ & $3.3 \pm 0.0$ & $12.5 \pm 0.0$ & $23.1 \pm 0.0$ & $28.3 \pm 0.0$ & $15.5 \pm 0.0$ & $11.8 \pm 0.0$ & $2.6 \pm 0.0$ & $1.3 \pm 0.0$ & 1 \\
\hline subsp. hispida & $0.2 \pm 0.0$ & $3.7 \pm 0.7$ & $17.0 \pm 3.6$ & $23.4 \pm 2.8$ & $27.8 \pm 2.1$ & $13.6 \pm 2.6$ & $10.0 \pm 2.8$ & $2.2 \pm 1.3$ & $1.1 \pm 0.7$ & 6 \\
\hline subsp. nevadensis & $0.2 \pm 0.0$ & $3.6 \pm 0.2$ & $15.5 \pm 1.3$ & $22.5 \pm 0.1$ & $34.9 \pm 4.8$ & $12.2 \pm 1.6$ & $7.4 \pm 2.5$ & $2.3 \pm 1.7$ & $0.6 \pm 0.5$ & 2 \\
\hline
\end{tabular}

R: resto; n: número de poblaciones estudiadas. 